\section{POSTNATAL VARIABLE HYPEROXIA SUPPRESSES MYELIN BASIC PROTEIN MRNA EXPRESSION INDUCED BY ANTENATAL LIPOPOLYSACCHARIDE}

E. Pilley ${ }^{1}$, J. Wade ${ }^{1}$, T. Gillespie ${ }^{2}$, I. Laing ${ }^{1}$, J.-C. Becher ${ }^{1}$, Jennifer Brown Research Group

${ }^{1}$ Jennifer Brown Research Group, Department of Neonatology, QMRI, University of Edinburgh,

2IMPACT Confocal Facility, University of Edinburgh, Edinburgh, UK

Background: Epidemiological and experimental studies show that hyperoxia and infection are independently associated with injury to the developing brain. Moreover inflammation may precondition the fetal brain resulting in increased or decreased injury when exposed to a postnatal insult. We examined the combined effects of antenatal bacterial endotoxin and postnatal variable hyperoxia on the immature rat brain.

Methods: Pregnant rats were injected intraperitoneally on E18 and E19 with lipopolysaccharide $(225 \mathrm{mcg} / \mathrm{kg})$ or saline. Dams and their pups were reared in room air or fluctuating hyperoxia (circa $10 \mathrm{kPa}$ ). Pup brains were examined at $\mathrm{P} 7$ for myelin basic protein (MBP) in the internal and external capsules using immunohistochemistry. Quantification of MBP mRNA was also undertaken.

Results: In the group exposed to antenatal lipopolysaccharide alone immunohistochemistry showed a decrease in MBP at P7 but there was a marked increase in MBP mRNA expression. Pups exposed to both antenatal lipopolysaccharide and postnatal variable hyperoxia showed a further decrease in MBP (immunohistochemistry). MBP mRNA expression was also markedly suppressed. Postnatal variable hyperoxia had little effect on myelination at P7 (immunohistochemistry) but MBP mRNA was decreased.

Conclusion: These results show that antenatal inflammation reduces myelination in the motor tracts of the developing brain. The increase in MBP mRNA production suggests that hypomyelination induced by endotoxin may be transient and potentially reversible. Postnatal variable hyperoxia has a synergistic effect in depressing myelination further and this prolonged insult results in suppression of mRNA expression. Where preterm infants are exposed to antenatal inflammation, hyperoxia may continue to disrupt myelination postnatally.
EARLY GENE REGULATION IN RETINA AND CHOROID AFTER ASHPYXIA AND RESUSCITATION IN NEWBORN PIGLETS

R. Solberg ${ }^{1}$, A. Arduini ${ }^{2}$, J. Escobar ${ }^{2}$, J. Sastre $^{2}$, O.D. Saugstad ${ }^{1}$, M. Vento ${ }^{3}$

${ }^{1}$ Department of Paediatric Research, Oslo University Hospital, Rikshospitalet, Oslo, Norway,

${ }^{2}$ Departamento de Fisiologia, Universidad de Valencia, ${ }^{3}$ Servicio de Neonatologia, Neonatologia Hospital, Universario Materno Infantil La Fe, Valencia, Spain

Background and aims: Low oxygen level and reactive oxygen species promote stabilization of HIF-1 $1 \alpha$, which in turn increases the expression of genes involved in angiogenesis (VEGFa , VEGFR1) and metabolism (GAPDH, ALDOC, PGK1). VEGFa and VEGFR1 play a crucial role in the neovascularization of the retina. VEGFR2 mediates the major cellular responses induced by VEGF, and also plays a role in neuroprotection.

Methods: Hypoxia was induced in newborn piglets. Retina and choroidea were obtained at end-hypoxia or after 15 min resuscitation with $21 \%$ or $100 \%$ oxygen. Control animals were kept anesthetized and ventilated. Gene expression of VEGFa, VEGFR1, VEGFR2, BDNF, ALDOC, GAPDH, PGK1 and HIF$1 \alpha$ was determined in retina and choroidea by RTPCR.

Results: There was a two-threefold increase in Vegfa expression in both retina and choroidea during hypoxia and after 15 min reoxygenation ( $p<0.01$ vs control). Vegfr2 expression was decreased by $50 \%$ $(p<0.05$ vs control) in retina and choroidea. Aldoc decreased by $75 \%$ in choroid $(p<0.001)$ and $25 \%$ in retina $(p<0.05)$ after hypoxia and resuscitation. Pgk1 was increased by $25 \%$ just in retina $(p<0.05$ vs control). Hif-1a mRNA level was not changed after hypoxia or resuscitation, as also observed for Vegfr1, Gapdh, Pgk1(in choroidea) and Bdnf ( $p>0.05$ vs control).

Conclusions: Low $\mathrm{PO}_{2}$ is responsible for adaptive responses both in retina and choroidea. The increase in the angiogenic factor VEGFa was not paralleled by the increase in other HIF-1 target genes. Repression of ALDOC may have important physiological significance, possibly to enhance the antioxidant defense. 